\title{
CONOCIMIENTOS Y PRÁCTICAS DE ESTUDIANTES SOBRE SUSTANCIAS PSICOACTIVAS EN UNA UNIVERSIDAD DE BARRANQUILLA
}

\author{
Damar Barraza Ospino ${ }^{1}$, Gloria Lastre Amell ${ }^{2}$
}

\section{Resumen}

Introducción. Las sustancias psicoactivas son compuestos de origen natural o sintético que al introducirse por vía oral, nasal, intramuscular o intravenosa ejercen un efecto directo sobre el sistema nervioso central. La importancia del estudio radicó en abordar a la población universitaria frente a los conocimientos y prácticas de consumo de sustancias psicoactivas. Método. Estudio cuantitativo, descriptivo, transversal, con estudiantes del área de la salud de una universidad privada de Barranquilla, en el año 2016. El universo fue de 4535 estudiantes de diferentes programas y la muestra de 324 estudiantes. El análisis estadístico se realizó en tablas frecuencias y porcentajes. Resultados. La muestra estudiada no tenía conocimiento sobre las sustancias psicoactivas, el $61 \%$ indicó que el alcohol es estimulante. El 91\% manifestó que las sustancias psicoactivas son perjudiciales para la salud. La sustancia de mayor consumo fue el alcohol (38\%), seguida por el tabaco (5\%) y finalmente, la marihuana (2\%). La población en estudio consume por curiosidad (61\%), por divertirse $(61 \%)$, por rumbear (59\%), por problemas familiares $(60 \%)$ o para enfrentar la soledad y la depresión (59\%). Conclusiones. los estudiantes no tiene conocimientos sobre la clasificación de las sustancias y las prácticas de consumo son sustancias lícitas.

Palabras clave: drogas ilícitas, consumidores de drogas, psicotrópicos,

conocimiento.

'Enfermera. Magíster en Salud Ocupacional. Docente del Programa de Enfermería, Universidad Metropolitana. damar.barraza@unimetro.edu.co

2 Enfermera. Especialista en Auditoría. Magíster en Salud Publica. Docente Programa de Enfermería, Universidad Metropolitana, Barranquilla y Universidad Simón Bolívar, Barranquilla.glastre2@unimetro.edu.co; glastre@unisimonbolivar. edu.co 


\title{
STUDENTS KNOWLEDGE AND PRACTICES ABOUT PSYCHOACTIVE SUBSTANCES IN A UNIVERSITY OF BARRANQUILLA
}

\author{
Damar Barraza Ospino, Gloria Lastre Amell
}

\section{Abstract}

Introduction. Psychoactive substances are compounds of natural or synthetic origin that, when are introduced orally, nasally, intramuscularly or intravenously cause a direct effect on the central nervous system. The relevance of the study lies on the fact of inquiring university population about the knowledge and practices of psychoactive substance use. Method. Quantitative, descriptive, cross-sectional study, with students from the health area of a private university of Barranquilla, in 2016. The population was 4535 students from different programs and the sample was taken from 324 students. The statistical analysis was carried out in frequencies charts and percentages. Results. The sample studied had any knowledge about psychoactive substances, $61 \%$ indicated that alcohol is stimulating. $91 \%$ said that psychoactive substances are harmful to health. The substance with the highest consumption was alcohol (38\%), followed by tobacco (5\%) and finally, marijuana (2\%). The population studied consumes because of curiosity (61\%), to have fun (61\%), partying (59\%), family issues (60\%) or to face loneliness and depression (59\%). Conclusions. Students do not have knowledge about the classification of substances and consumption practices are licit substances. 


\section{CONHECIMENTO E PRÁTICAS DE ALUNOS SOBRE SUBSTÂNCIAS PSICOATIVAS EM UMA UNIVERSIDADE DE BARRANQUILLA}

Damar Barraza Ospino, Gloria Lastre Amell

\section{Resumo}

Introdução. As substâncias psicoativas são compostos de origem natural ou sintética que, quando introduzidos por via oral, nasal, intramuscular ou intravenosa, exercem um efeito direto sobre o sistema nervoso central. O estudo é importante em quanto que aborda a população universitária em relação aos conhecimentos e práticas do uso de substâncias psicoativas. Método. Estudo quantitativo, descritivo, transversal, com estudantes da área da saúde de uma universidade privada de Barranquilla, em 2016. O universo foi de 4535 alunos de diferentes programas e a amostra de 324 alunos. A análise estatística foi realizada em tabelas, frequências e porcentagens. Resultados. A amostra estudada não tinha conhecimento sobre substâncias psicoativas, $61 \%$ indicaram que o álcool é estimulante. $91 \%$ disseram que as substâncias psicoativas são prejudiciais à saúde. A substância com maior consumo foi o álcool (38\%), seguida do tabaco (5\%) e, finalmente, a maconha (2\%). O consumo da população do estudo é devido à curiosidade (61\%), diversão (61\%), festejos (59\%), problemas familiares $(60 \%)$ ou para enfrentar a solidão e a depressão (59\%). Conclusões. Os alunos não têm conhecimento sobre a classificação de substâncias e práticas de consumo com substâncias ilícitas. 


\section{Introducción}

La Organización Mundial de la Salud define droga como "toda sustancia que, introducida en un organismo vivo, pueda alterar una o varias de sus funciones" $(1,2)$. Reiterando este concepto, toda sustancia de origen natural o sintético que al introducirse por cualquier vía, ejerce un efecto directo sobre el sistema nervioso central (SNC), ocasionando cambios específicos a sus funciones $y$ alterando todas sus dimensiones: psicológica, fisiológica, entre otras (3).

El estudio nacional de sustancias psicoactivas indica que el $0,5 \%$ y $1 \%$ de la población adulta es adicta a los opiáceos morfina, heroína; entre $5 \%$ al $10 \%$ al alcohol y entre un $30 \%$ al $40 \%$, al tabaco (4). Así mismo, la prevalencia del consumo de sustancias ilícitas en el grupo de 18 a 24 años, fue de $8,7 \%$, los adolescentes de $4.8 \%$, y las personas de 25 a 34 años, con una prevalencia del $4,3 \%$. Se considera que los mayores consumidores son los adultos jóvenes de todas las sociedades, la prevalencia disminuye con la edad (maduración, obligaciones sociales), siendo frecuente en varones (5).

La Oficina de las Naciones Unidas contra la Droga y el Delito (UNODC) indicó que en el año 2017, un total de 246 millones de personas, es decir una de cada 20 personas de edades comprendidas entre los 15 y 64 años, consumieron drogas ilícitas (6). En América y Europa, el consumo de cocaína ha disminuido en su conjunto, el consumo de cannabis y el uso no médico de opioides farmacéuticos ha seguido en aumento, en cuanto al consumo de estimulantes de tipo anfeta- mínico varía de una región a otra, y en algunas subregiones, como Asia sudoriental se ha evidenciadoun aumento del consumo de metanfetamina (7).

El Informe Mundial sobre las Drogas 2015 expresó escasa información sobre la prevalencia mundial del consumo de drogas. En los opioides fue de $0,7 \%$ de la población adulta y el consumo de opiáceos de $0,4 \%$, correspondiente a $32,4 \mathrm{y}$ 16,5 millones de personas respectivamente. La prevalencia del consumo de esta sustancia sigue siendo elevada en América del Norte (3,8\%). En los Estados Unidos hay indicios de un desplazamiento parcial del consumo de opioides hacia el consumo de heroína. En 2014, las nuevas sustancias psicoactivas notificadas fueron los cannabinoides sintéti$\cos (39 \%)$, fenetilaminas (18\%) y catinonas sintéticas (15\%) (6).

La dimensión del problema sobre las drogas se hace evidente, debido a que 1 de cada 10 consumidores es un consumidor problemático, el cual sufre trastornos ocasionados por el consumo de las sustancias psicoactivas. En el país de Malasia 27 millones de personas de la población son consumidores problemáticos; 12,19 millones de personas consumen drogas inyectables (6).

A nivel nacional el Ministerio del Interior y de Justicia, la Dirección Nacional de Estupefacientes y el Ministerio de la Protección Social, con el apoyo de la Oficina de Naciones Unidas contra las Drogas y el Delito, la Embajada de Estados Unidos en Colombia y la Comisión Interamericana para el Control del Abuso de Drogas (CICAD) de la Organización de Estados Americanos (OEA), realizaron un estu- 
dio en 126 municipios de los 32 departamentos colombianos, donde mostraron que en el departamento antioqueño y la zona occidental, constituida por Valle, Cauca, Nariño y Bogotá, eran las regiones del país donde hubo mayor consumo de drogas ilícitas (7).

El Observatorio de Drogas de Colombia indicó que el consumo de drogas está creciendo en el país, debido a que no solamente cada vez más personas consumen drogas como lo evidencian los estudios, sino, porque el fenómeno mundial de las drogas emergentes es también una realidad en el contexto nacional en el que cada vez surgen sustancias diferentes, que hacen más diverso el mercado ilegal de drogas. Considerando que Colombia no es ajena a este problema, debido a su impacto y repercusión que tiene en el campo de la salud pública, como lo reconoce en el Artículo 32 de la Ley 1122 de 2007 (8), con referencia a la población universitaria, según los estudios comparativos realizados en los países andinos, Colombia tiene los consumos más altos en la mayoría de sustancias (9).

En los estudios de Navia-Bueno y Cols, Wright, Takayanagui y Ventura, se demostró que los estudiantes universitarios no pueden clasificar las sustancias adictivas según su uso socio legal, el $82 \%$ reconoció que sí son drogas y psicotrópicas. Se puede observar en cuanto al conocimiento del fenómeno de las drogas, que este se presenta de manera diferenciada en las categorías de los individuos, así como en los diferentes niveles universitarios, siendo muy variado, lo que indica la necesidad de mantener la enseñanza alrededor de la integración, acción y conocimientos referentes a las drogas y a las políticas y programas a nivel nacional e internacional $(10,11)$.

En estudios realizados acerca del conocimiento sobre el consumo de sustancias psicoactivas en estudiantes de enfermería, se evidenció que el $81 \%$ de los estudiantes era de sexo femenino, y sus edades comprendían entre 17 y 21 años, el 99,6\% consideró que el consumo de sustancias psicoactivas son perjudiciales, en tanto que el $95 \%$ estimó que el cerebro es el órgano más afectado por el consumo. El 9,5\% consideró que el alcohol no es una droga depresora, y el $18 \%$ afirmó que la heroína tampoco no es sustancia depresora. Las benzodiacepinas constituyen la sustancia que en mayor proporción (83\%) fue seleccionada como estimulante $(12,14)$.

Por lo anterior, este estudio es importante debido a la necesidad de conocer de dónde viene el problema y abordar de forma específica la población universitaria, porque en esta etapa es donde los estudiantes se enfrentan a un riesgo mayor de consumo de sustancias psicoactivas (SPA); esto se ve influenciado en el periodo de evolución del universitario como es la búsqueda de identidad, autonomía y la necesidad de experimentar nuevas sensaciones en su vida y ser recenonocidos ante su grupo.

La política pretende ofrecer lineamientos que permitan identificar, comprender y contrarrestar los factores sociales $y$ personales que inciden en el consumo de SPA y además, guiar el diseño de planes y programas a nivel territorial que respondan a las necesidades de la 
en materia de prevención, superación de los riesgos y daños asociados a estas sustancias. Es por ello que el objetivo de este estudio fue determinar los conocimientos y prácticas de estudiantes sobre sustancias psicoactivas en una universidad de Barranquilla.

\section{Método}

El estudio fue de tipo cuantitativo, descriptivo, transversal, con estudiantes del área de la salud de una universidad privada de Barranquilla. De un universo de 4535 estudiantes de los programas de enfermería, medicina, fisioterapia, trabajo social, nutrición, odontología, psicología, terapia ocupacional, bacteriología, fonoaudiología, optometría de todos los semestres académicos (Tabla 1). Se trabajó con una muestra de 324 estudiantes, teniendo en cuenta que la muestra fue de 354 personas, pero se desecharon 30 encuestas por no alcanzar la totalidad de las respuestas, controlando el sesgo de información. Así mismo, se realizó un muestreo probabilístico aleatorio simple, a cargo de un profesional estadístico, con el fin de definir la población a estudiar y así, evitar sesgo de selección.

Para la recolección de la información se

Tabla 1. Cálculo muestral.

\begin{tabular}{l|c|c|c|}
\hline \multirow{2}{*}{ Programa } & Cantidad & Warm & \multirow{2}{*}{ Hot } \\
\cline { 3 - 3 } & & \% & \\
\hline Medicina & 2474 & $55 \%$ & 193 \\
Enfermería & 574 & $13 \%$ & 45 \\
Odontología & 515 & $11 \%$ & 40 \\
Nutrición & 373 & $8 \%$ & 29 \\
Psicología & 202 & $4 \%$ & 16 \\
Optometría & 129 & $3 \%$ & 10 \\
Fisioterapia & 103 & $2 \%$ & 8 \\
Bacteriología & 67 & $1 \%$ & 5 \\
Trabajo Social & 50 & $1 \%$ & 4 \\
Terapia Ocupacional & 48 & $1 \%$ & 4 \\
TOTAL & 4535 & & \\
\hline
\end{tabular}


aplicó una encuesta titulada "Factores predisponentes y asociados al consumo de sustancias psicoactivas", cuyo autor es Cepeda Díaz, Aldana y Ossío, la cual consta de 33 preguntas, organizadas en tres secciones: socio-demográficos (11 preguntas), conocimientos (4), y prácticas (18). Esta constó de preguntas de selección múltiple, preguntas cerradas de Si, No, No sé, si está en desacuerdo, totalmente en acuerdo y desacuerdo.

Previo a la recolección de la información, se realizó una prueba piloto con 30 estudiantes para determinar la comprensión por parte de los encuestadores y el tiempo empleado para su diligenciamiento para obtener la validez y confiabilidad del instrumento.

Así mismo, se estableció el contacto con los diferentes programas de la universidad privada de Barranquilla, solicitando la autorización con los directores de cada programa. Posteriormente, los investigadores se dirigieron a cada una de las aulas de clase, socializando el consentimiento informado y dando a conocer las consideraciones éticas que se tendrían en cuenta. Además, se les explicó cómo diligenciar la encuesta y el tiempo de aplicación, el cual fue de 15 minutos. Una vez informado el proceso a la población se realizó la encuesta. Para llevar a cabo la actividad, cada uno de los investigadores se dividió los semestres y programas, realizando la recolección de la información, en un periodo de dos meses.

Después se procedió a diseñar la matriz en Excel, registrándose las variables significativas, posteriormente los datos fueron procesados con SPSS versión 15.0.

Según el análisis estadístico, en este estudio descriptivo se tuvieron en cuenta las variables cualitativas, las cuales fueron medidas en tablas de frecuencia y porcentaje. Los criterios de inclusión fueron que los estudiantes estuviesen matriculados en el primer periodo de 2016, en todos los programas del área de la salud y los criterios de exclusión fueron estudiantes con alguna limitación física o incapacidad médica que no les permitiera realizar la encuesta. Se tuvo en cuenta la Resolución 008430 de 1993 (15), que establece las normas científicas, técnicas y administrativas para la investigación en salud.

\section{Resultados}

Las variables sociodemográficas de los estudiantes de la universidad privada de Barranquilla donde se realizó el estudio acerca del conocimiento y prácticas de las sustancias lícitas e ilícitas en el consumo de SPA, señalan que la mayoría de la población estudiada es procedente de Barranquilla, seguido de los municipios de Soledad y Sabanalarga, el género que predominó fue el femenino, el estado civil, solteros de estrato socioeconómico 2 y 3 y la preferencia sexual fue heterosexual (Tabla 2.) 
INVESTIGACIONES ANDINA No. 36, Vol. 20

Tabla 2. Aspectos sociodemográficos de los estudiantes de la universidad privada de Barranquilla.

\begin{tabular}{|c|c|c|}
\hline \multicolumn{3}{|c|}{ ASPECTOS SOCIODEMOGRÁFICOS } \\
\hline LUGAR DE RESIDENCIA & FRECUENCIA & $\%$ \\
\hline Baranoa & 1 & $0 \%$ \\
\hline Barranquilla & 278 & $86 \%$ \\
\hline Galapa & 1 & $0 \%$ \\
\hline Juan De Acosta & 1 & $0 \%$ \\
\hline Malambo & 1 & $0 \%$ \\
\hline Manatí & 1 & $0 \%$ \\
\hline Palmar & 2 & $1 \%$ \\
\hline Polonuevo & 1 & $0 \%$ \\
\hline Ponedra & 2 & $1 \%$ \\
\hline Puerto Colombia & 2 & $1 \%$ \\
\hline Riohacha & 1 & $0 \%$ \\
\hline Sabanagrande & 1 & $0 \%$ \\
\hline Sabanalarga & 10 & $3 \%$ \\
\hline Salgar & 1 & $0 \%$ \\
\hline Soledad & 19 & $6 \%$ \\
\hline Universal & 1 & $0 \%$ \\
\hline Zapayan & 1 & $0 \%$ \\
\hline TOTAL & 324 & $100 \%$ \\
\hline GÉNERO & FR & $\%$ \\
\hline Femenino & 190 & $59 \%$ \\
\hline Masculino & 134 & $41 \%$ \\
\hline TOTAL & 324 & $100 \%$ \\
\hline PREFERENCIA SEXUAL & FR & $\%$ \\
\hline Homosexual & 10 & $3 \%$ \\
\hline Heterosexual & 312 & $96 \%$ \\
\hline Bisexual & 2 & $1 \%$ \\
\hline TOTAL & 324 & $100 \%$ \\
\hline NÚMERO DE HERMANOS & FR & $\%$ \\
\hline 0 & 15 & $5 \%$ \\
\hline 1 & 61 & $19 \%$ \\
\hline 2 & 94 & $29 \%$ \\
\hline 3 & 74 & $23 \%$ \\
\hline 4 & 47 & $15 \%$ \\
\hline 5 & 24 & $7 \%$ \\
\hline 6 & 8 & $2 \%$ \\
\hline
\end{tabular}




\begin{tabular}{ccc}
7 & 1 & $0 \%$ \\
TOTAL & 324 & $100 \%$ \\
ESTRATO SOCIAL & FR & $\%$ \\
1 & 58 & $18 \%$ \\
2 & 110 & $34 \%$ \\
4 & 92 & $28 \%$ \\
5 & 50 & $15 \%$ \\
6 & 10 & $3 \%$ \\
TOTAL & 4 & $1 \%$ \\
ESTADO CIVIL & 324 & $100 \%$ \\
Soltero & FR & $\%$ \\
Union libre & 311 & $96 \%$ \\
Casado & 7 & $2 \%$ \\
Separado & 5 & $2 \%$ \\
TOTAL & 1 & $0 \%$ \\
\hline
\end{tabular}

Fuente: Encuesta.

Con respecto a la variable conocimiento sobre el consumo de SPA de los estudiantes de acuerdo con su clasificación, la mayoría respondió que el alcohol era estimulante. Por otro lado, clasifican las anfetaminas, nicotina, cocaína, éxtasis y el bazuco como drogas estimulantes. Con relación a las benzodiacepinas, los estudiantes respondieron en mayor porcentaje que son drogas depresoras, seguido del alcohol, marihuana y los inhalables; así mismo, se observa en la Tabla 3 que los estudiantes categorizan al éxtasis, LSD y la marihuana como sustancias alucinógenas.

Tabla 3. Conocimiento sobre la clasificación de las sustancias psicoactivas.

\begin{tabular}{c|c|c|c|c|c|c|c|c}
\hline \multicolumn{7}{|c|}{ CONOCIMIENTO SOBRE LA CLASIFICACION DE LAS SUSTANCIAS PSICOACTIVAS } \\
\hline CONOCIMIENTO & $\begin{array}{c}\text { Estimu- } \\
\text { lante }\end{array}$ & $\%$ & $\begin{array}{c}\text { Depre- } \\
\text { siva }\end{array}$ & $\%$ & $\begin{array}{c}\text { Aluci- } \\
\text { nógena }\end{array}$ & $\%$ & $\begin{array}{c}\text { No } \\
\text { tengo } \\
\text { conoci- } \\
\text { miento }\end{array}$ & Total \\
\hline Alcohol & 198 & $61 \%$ & 64 & $20 \%$ & 14 & $4 \%$ & 48 & 324 \\
Anfetaminas & 66 & $20 \%$ & 53 & $16 \%$ & 107 & $33 \%$ & 98 & 324 \\
Éxtasis & 69 & $21 \%$ & 25 & $8 \%$ & 152 & $47 \%$ & 78 & 324 \\
Lsd & 41 & $13 \%$ & 34 & $10 \%$ & 125 & $39 \%$ & 124 & 324
\end{tabular}


INVESTIGACIONES ANDINA No. 36, Vol. 20

$\begin{array}{ccccccccc}\text { Benzodiacepina } & 41 & 13 \% & 106 & 33 \% & 52 & 16 \% & 125 & 324 \\ \text { Orlasteina } & 35 & 11 \% & 41 & 13 \% & 57 & 18 \% & 191 & 324 \\ \text { Heroína } & 63 & 19 \% & 42 & 13 \% & 145 & 45 \% & 74 & 324 \\ \text { Cocaina } & 107 & 33 \% & 29 & 9 \% & 116 & 36 \% & 72 & 324 \\ \text { Marihuana } & 89 & 27 \% & 54 & 17 \% & 124 & 38 \% & 57 & 324 \\ \text { Nicotina(Cigarrilo) } & 137 & 42 \% & 53 & 16 \% & 59 & 18 \% & 75 & 324 \\ \text { Inhalantes(Boxer) } & 60 & 19 \% & 49 & 15 \% & 130 & 40 \% & 85 & 324 \\ \text { Bazuco } & 62 & 19 \% & 47 & 15 \% & 128 & 40 \% & 87 & 324\end{array}$

Fuente: Encuesta.

Tabla 4. Conocimiento sobre efecto perjudicial de las sustancias psicoactivas para la salud.

\begin{tabular}{l|c|c|c|c|c|c|c|}
\hline \multicolumn{6}{c}{ LAS SUSTANCIAS PSICOACTIVAS PRODUCEN ALGÚN EFECTO PERJUDICIAL } \\
PARA LA SALUD \\
\hline \multicolumn{1}{c}{ SUSTANCIA } & SÍ & $\%$ & NO & $\%$ & NO SE & $\%$ & TOTAL \\
\hline Alcohol & 306 & $94 \%$ & 14 & $4 \%$ & 4 & $1 \%$ & 324 \\
Anfetaminas & 300 & $93 \%$ & 6 & $2 \%$ & 18 & $6 \%$ & 324 \\
Éxtasis & 312 & $96 \%$ & 4 & $1 \%$ & 8 & $2 \%$ & 324 \\
LSD & 288 & $89 \%$ & 6 & $2 \%$ & 30 & $9 \%$ & 324 \\
Benzodiacepina & 258 & $80 \%$ & 24 & $7 \%$ & 42 & $13 \%$ & 324 \\
Orlasteina & 240 & $74 \%$ & 12 & $4 \%$ & 72 & $22 \%$ & 324 \\
Heroína & 315 & $97 \%$ & 3 & $1 \%$ & 6 & $2 \%$ & 324 \\
Cocaína & 316 & $98 \%$ & 4 & $1 \%$ & 4 & $1 \%$ & 324 \\
Marihuana & 315 & $97 \%$ & 4 & $1 \%$ & 5 & $2 \%$ & 324 \\
Nicotina(Cigarrilo) & 311 & $96 \%$ & 8 & $2 \%$ & 5 & $2 \%$ & 324 \\
Inhalantes(Boxer) & 311 & $96 \%$ & 8 & $2 \%$ & 5 & $2 \%$ & 324 \\
Bazuco & 315 & 34 & $10 \%$ & 7 & $2 \%$ & 324 \\
\hline
\end{tabular}

64

Fuente: Encuesta.

Nota: La mayoría de los estudiantes manifestó que las drogas son sustancias perjudiciales para la salud. 
Tabla 5. Conocimiento sobre órganos del cuerpo que se pueden afectar por el consumo de drogas.

\begin{tabular}{c|c|c|c|c|c}
\hline \multicolumn{5}{c}{ ÓRGANOS DEL CUERPO QUE SE PUEDEN AFECTAR POR EL CONSUMO DE DROGAS } \\
\hline Criterios & $\mathrm{Fr}$ & $\%$ & Criterios & $\mathrm{Fr}$ & $\%$ \\
\hline Cerebro & 315 & $97 \%$ & Pulmón & 300 & $93 \%$ \\
Sangre & 222 & $69 \%$ & Riñón & 247 & $76 \%$ \\
$\begin{array}{c}\text { Estómago e } \\
\text { Intestinos }\end{array}$ & 168 & $52 \%$ & Ojos & 219 & $68 \%$ \\
Corazón & 252 & $78 \%$ & Otros... ¿Cuál? & & $0 \%$ \\
\hline
\end{tabular}

Fuente: Encuesta

Nota: De acuerdo con la variable si las sustancias lícitas e ilícitas afecta algún órgano del cuerpo, la población expresó que los órganos que mayormente se afectan son el cerebro y los pulmones, seguido del corazón, los riñones, la sangre y los ojos.

Tabla 6. Práctica de consumo de drogas.

\begin{tabular}{|c|c|c|c|c|c|c|c|c|c|c|c|}
\hline Criterios & Nunca & $\%$ & $\begin{array}{l}\text { solo } \\
\text { una } \\
\text { vez }\end{array}$ & $\%$ & $\begin{array}{l}\text { actual- } \\
\text { mente }\end{array}$ & $\%$ & $\begin{array}{c}\text { Las } \\
\text { consumí } \\
\text { frecuente- } \\
\text { mente, pero } \\
\text { actualmen- } \\
\text { te no las } \\
\text { consumo }\end{array}$ & $\%$ & $\begin{array}{c}\text { Solo } \\
\text { las } \\
\text { con- } \\
\text { sumí } \\
\text { oca- } \\
\text { sional- } \\
\text { mente }\end{array}$ & $\%$ & Total \\
\hline Alcohol & 69 & $21 \%$ & 34 & $10 \%$ & 123 & $38 \%$ & 20 & $6 \%$ & 78 & $24 \%$ & 324 \\
\hline Anfetaminas & 307 & $95 \%$ & 12 & $4 \%$ & 3 & $1 \%$ & 0 & $0 \%$ & 2 & $1 \%$ & 324 \\
\hline Éxtasis & 309 & $95 \%$ & 10 & $3 \%$ & 3 & $1 \%$ & 0 & $0 \%$ & 2 & $1 \%$ & 324 \\
\hline LSD & 310 & $96 \%$ & 12 & $4 \%$ & 0 & $0 \%$ & 0 & $0 \%$ & 2 & $1 \%$ & 324 \\
\hline Benzodiacepinas & 303 & $94 \%$ & 13 & $4 \%$ & 3 & $1 \%$ & 2 & $1 \%$ & 3 & $1 \%$ & 324 \\
\hline Orlasteina & 315 & $97 \%$ & 8 & $2 \%$ & 0 & $0 \%$ & 0 & $0 \%$ & 1 & $0 \%$ & 324 \\
\hline Heroína & 313 & $97 \%$ & 8 & $2 \%$ & 2 & $1 \%$ & 0 & $0 \%$ & 1 & $0 \%$ & 324 \\
\hline Cocaína & 313 & $97 \%$ & 5 & $2 \%$ & 4 & $1 \%$ & 0 & $0 \%$ & 2 & $1 \%$ & 324 \\
\hline Marihuana & 286 & $88 \%$ & 23 & $7 \%$ & 8 & $2 \%$ & 3 & $1 \%$ & 4 & $1 \%$ & 324 \\
\hline $\begin{array}{l}\text { Nicotina } \\
\text { (Cigarrillo) }\end{array}$ & 275 & $85 \%$ & 23 & $7 \%$ & 15 & $5 \%$ & 8 & $2 \%$ & 3 & $1 \%$ & 324 \\
\hline $\begin{array}{c}\text { Inhalantes } \\
\text { (Boxer) }\end{array}$ & 311 & $96 \%$ & 11 & $3 \%$ & 0 & $0 \%$ & 0 & $0 \%$ & 2 & $1 \%$ & 324 \\
\hline Bazuco & 311 & $96 \%$ & 10 & $3 \%$ & 2 & $1 \%$ & 0 & $0 \%$ & 1 & & 324 \\
\hline
\end{tabular}

Nota: Los estudiantes expresaron que la mayoría de sustancias que consumen son lícitas como es el alcohol y tabaco, seguido de drogas ilícitas como la marihuana. 
Tabla 7. Razones por las cuales la gente consume o puede llegar a consumir drogas.

\begin{tabular}{|c|c|c|c|c|c|c|c|c|c|c|c|}
\hline Criterios & $\begin{array}{l}\text { Total- } \\
\text { mente } \\
\text { de } \\
\text { acuerdo }\end{array}$ & $\%$ & $\begin{array}{c}\text { De } \\
\text { acuerdo } \\
\text { en parte }\end{array}$ & $\%$ & $\begin{array}{c}\text { No } \\
\text { tengo } \\
\text { opinión }\end{array}$ & $\%$ & $\begin{array}{l}\text { Des- } \\
\text { acuerdo } \\
\text { en parte }\end{array}$ & $\%$ & $\begin{array}{l}\text { Total- } \\
\text { mente } \\
\text { en des- } \\
\text { acuerdo }\end{array}$ & $\%$ & Total \\
\hline $\begin{array}{l}\text { Para poder } \\
\text { relacionarme más } \\
\text { fácilmente con la } \\
\text { gente }\end{array}$ & 75 & $23 \%$ & 85 & $26 \%$ & 58 & $15 \%$ & 24 & $7 \%$ & 82 & $25 \%$ & 324 \\
\hline $\begin{array}{l}\text { Para satisfacer la } \\
\text { curiosidad acerca } \\
\text { de sus efectos }\end{array}$ & 90 & $28 \%$ & 107 & $33 \%$ & 50 & $15 \%$ & 31 & $10 \%$ & 46 & $14 \%$ & 324 \\
\hline Para divertirse & 111 & $34 \%$ & 88 & $27 \%$ & 49 & $15 \%$ & 21 & $6 \%$ & 55 & $17 \%$ & 324 \\
\hline Para rumbear & 99 & $31 \%$ & 91 & $28 \%$ & 62 & $19 \%$ & 20 & $6 \%$ & 52 & $16 \%$ & 324 \\
\hline $\begin{array}{l}\text { Para imitar a } \\
\text { alguien }\end{array}$ & 73 & $23 \%$ & 83 & $26 \%$ & 73 & $23 \%$ & 21 & $6 \%$ & 74 & $23 \%$ & 324 \\
\hline $\begin{array}{l}\text { Por influencia de } \\
\text { mis amigos }\end{array}$ & 97 & $30 \%$ & 83 & $26 \%$ & 58 & $18 \%$ & 26 & $8 \%$ & 60 & $19 \%$ & 324 \\
\hline $\begin{array}{l}\text { Para ser aceptado } \\
\text { en un grupo }\end{array}$ & 89 & $27 \%$ & 90 & $28 \%$ & 56 & $17 \%$ & 22 & $7 \%$ & 67 & $21 \%$ & 324 \\
\hline $\begin{array}{l}\text { Por ser algo } \\
\text { prohibido }\end{array}$ & 69 & $21 \%$ & 84 & $26 \%$ & 71 & $22 \%$ & 29 & $9 \%$ & 71 & $22 \%$ & 324 \\
\hline $\begin{array}{l}\text { Para enfrentar la } \\
\text { depresión }\end{array}$ & 107 & $33 \%$ & 85 & $26 \%$ & 60 & $19 \%$ & 20 & $6 \%$ & 52 & $16 \%$ & 324 \\
\hline $\begin{array}{l}\text { Para enfrentar la } \\
\text { soledad }\end{array}$ & 100 & $31 \%$ & 90 & $28 \%$ & 62 & $19 \%$ & 18 & $6 \%$ & 54 & $17 \%$ & 324 \\
\hline $\begin{array}{l}\text { Por problemas } \\
\text { familiares }\end{array}$ & 117 & $36 \%$ & 78 & $24 \%$ & 55 & $17 \%$ & 21 & $6 \%$ & 53 & $16 \%$ & 324 \\
\hline $\begin{array}{l}\text { Por problemas } \\
\text { afectivos y/o } \\
\text { sentimentales }\end{array}$ & 104 & $32 \%$ & 83 & $26 \%$ & 61 & $19 \%$ & 23 & $7 \%$ & 53 & $16 \%$ & 324 \\
\hline $\begin{array}{l}\text { Por las sensacio- } \\
\text { nes que provoca }\end{array}$ & 97 & $30 \%$ & 92 & $28 \%$ & 58 & $18 \%$ & 22 & $7 \%$ & 55 & $17 \%$ & 324 \\
\hline $\begin{array}{c}\text { Para disipar el } \\
\text { estrés }\end{array}$ & 93 & $29 \%$ & 88 & $27 \%$ & 62 & $19 \%$ & 22 & $7 \%$ & 59 & $18 \%$ & 324 \\
\hline $\begin{array}{c}\text { Para expresar } \\
\text { independencia y a } \\
\text { veces hostilidad }\end{array}$ & 81 & $25 \%$ & 74 & $23 \%$ & 66 & $20 \%$ & 33 & $10 \%$ & 70 & $22 \%$ & 324 \\
\hline $\begin{array}{l}\text { Para rendir más } \\
\text { en el trabajo o } \\
\text { estudio }\end{array}$ & 75 & $23 \%$ & 70 & $22 \%$ & 68 & $21 \%$ & 31 & $10 \%$ & 80 & $25 \%$ & 324 \\
\hline $\begin{array}{l}\text { Para escapar de la } \\
\text { realidad }\end{array}$ & 121 & $37 \%$ & 69 & $21 \%$ & 52 & $16 \%$ & 21 & $6 \%$ & 61 & $19 \%$ & 324 \\
\hline $\begin{array}{l}\text { Para tener expe- } \\
\text { riencias nuevas, } \\
\text { placenteras, } \\
\text { emocionantes o } \\
\text { peligrosas }\end{array}$ & 112 & $35 \%$ & 86 & $27 \%$ & 51 & $16 \%$ & 17 & $5 \%$ & 58 & $18 \%$ & 324 \\
\hline
\end{tabular}

Fuente: Encuesta.

Nota: Se observa que la mayoría de los estudiantes respondió que los estudiantes consumen sustancias 66 lícitas e ilícitas por curiosidad, para divertirse y rumbear y por problemas familiares; otros motivos son para enfrentar la soledad y la depresión. 


\section{Discusión de resultados}

Al caracterizar a la población estudiada se evidenció que el género que predominó fue el femenino, con un 59\% y el $41 \%$ masculino, al comparar este resultado con la investigación de Lastre-Amell, et al., mostró que el $81 \%$ de los estudiantes era de género femenino y el $19 \%$, masculino (13).

La preferencia sexual de la población estuvo en $96 \%$ en ser heterosexual y en menor proporción homosexual con un $3 \%$ y bisexual $1 \%$; con respecto al estrato social se identificó que la mayoría pertenece a un estrato social $2(29 \%)$ y 3 (23\%), contrastando estos datos con Castellón, et al.,(14) se observó que el 99\% de los estudiantes son heterosexuales y el $1 \%$ se declaró homosexual, además se encontró que el $41.4 \%$ pertenece a estrato socioeconómico 2 , seguido del 3 con un $23,7 \%$; igualmente el informe final del estudio nacional de consumo de SPA reveló que el estrato con mayor cantidad de población fue el $2(38,3 \%)$ y el 25,7\% de la población representó en el estrato 1 (16).

Con respecto a la variable de conocimiento sobre el consumo de SPA de los estudiantes según su clasificación demostraron que el alcohol era estimulante, considerando que no tiene conocimiento claro de la clasificación de esta sustancias, debido a que esta droga pertenece a sustancias depresoras; en cambio otros si tienen conocimiento sobre que las anfetaminas, nicotina, cocaína, éxtasis y el bazuco son drogas estimulantes, resultados acordes con estudios encontrados, donde el "Conocimientos, actitudes y prácticas acerca del uso de las drogas en estudiantes indicó que en porcentaje no muy alto (9\%) demostró conocer acerca de las sustancias psicoactivas (9).

La investigación señalo que la mayoría de los estudiantes manifestaron que las drogas son sustancias perjudiciales para la salud, pero se sigue observando que hay población que no sabe si algunas de estas sustancias son perjudiciales, considerando que las drogas ilícitas son sustancias que afectan la salud del individuo. Por lo anterior, este estudio nos conlleva a fortalecer la política de la promoción de la salud y prevención de la enfermedad y a trabajar articuladamente con bienestar universitario o servicios de salud amigables con el fin de la mitigación de los daños en aquellos que han tenido contacto con las SPA o evitar el consumo de las sustancias.

De acuerdo con la variable si las sustancias licitas e ilícitas afecta algún órgano del cuerpo, los estudiantes expresaron que los órganos que mayormente se afectan son el cerebro y los pulmones, seguido de corazón, los riñones, la sangre y ojos; es decir que el consumo de estas sustancias, sí afectan los órganos del cuerpo, unos más que otros. Corroborando este resultado con Coullaut-Valera (17), donde la lucha contra el consumo, es caracterizar y cuantificar el daño que dicho abuso produce en el cerebro. Así mismo en otro estudio revelo que los estudiantes de enfermería de la universidad de Barranquilla se identificó que el cerebro, la sangre y los pulmones son aquellos órganos del cuerpo que más sufre los efectos de las sustancias psicoac- 
En la variable prácticas de consumo, la mayoría de los estudiantes expresó que la mayor parte de sustancias que consumen son lícitas como el alcohol y el tabaco, seguido de drogas ilícitas como la marihuana. De acuerdo con el estudio de Barbieri (18), se observó que el 20\% de los jóvenes informa consumir alcohol, por lo menos una vez a la semana, el $13 \%$ fuma por lo menos una vez al día y el $4 \%$ consume drogas ilegales (marihuana, cocaína, éxtasis, heroína, popper, entre otros alucinógenos). Estos datos son similares a los encontrados en la investigación, en la cual se evidenció que las sustancias de mayor consumo son el alcohol y tabaco, seguido de drogas ilícitas como la marihuana (19).

Se observó que la mayoría de los estudiantes respondió que consumen sustancias licitas e ilícitas por curiosidad, para divertirse y rumbear y por problemas familiares, otros motivos son para enfrentar la soledad y la depresión. A nivel etiológico, el consumo juvenil de sustancias psicoactivas responde tanto a intentos de satisfacción de motivaciones hedónicas como propiamente a afanes explorativos, prácticas ritualizadas, deseos de experimentación, desajustes socio afectivos, sobre activaciones varias y a necesidades percibidas de integración en el grupo de iguales y sus estilos de ocio juvenil, propios de una cultura recreativa (19). Hernández et al, (20), en su estudio indicaron que dentro de los factores de riesgo asociados al consumo de sustancias psicoactivas son la violencia intrafamiliar, el abuso, el maltrato, la presión de grupo, el fácil acceso a las drogas y la inactividad en el tiempo libre.

\section{Conclusiones}

Los estudiantes de los programas del área de la salud de la universidad privada no tienen conocimiento acerca de la clasificación de las sustancias psicoactivas, es decir ignoran si son depresoras, estimulantes o alucinógenas, entre otras; de acuerdo con las prácticas de consumo de sustancias psicoactivas, las que son lícitas tienen una mayor prevalencia en la comunidad universitaria.

Por lo anterior se concluye que la universidad debe fortalecer planes y acciones estratégicas en la temática de sustancias psicoactivas, que promuevan mejores prácticas de autocuidado en los estudiantes, desde una perspectiva de transformación cultural, educativa y comunicativa, además de la participación social y comunitaria para la prevención, atención y mitigación de los problemas, trastornos y eventos vinculados con la salud mental y al consumo de sustancias psicoactivas de acuerdo con lo manifestado en el Plan Decenal de Salud Pública, involucrando a estudiantes consumidores y no consumidores, alianzas entre las diferentes áreas de servicios de las universidades, directivos, administrativos, educadores profesionales, representantes estudiantiles y redes sociales, mediante capacitaciones, charlas de los docentes en sus clases o en espacios académicos, como eje transversal en el currículo para favorecer un estado de salud y bienestar. 


\section{Fuentes de financiación}

Este estudio fue financiado por la Universidad Metropolitana de Barranquilla.

\section{Conflicto de intereses}

Este estudio no tiene conflicto de intereses.

\section{Referencias}

1. Guía para el promotor de "Nueva Vida". Capítulo I. Conceptos básicos sobre adicciones. [Seriado en internet]. [Citado 10 marzo de 2017]. Disponible en: http:// www.conadic.salud.gob.mx/pdfs/nueva vida/prevad_cap1.pdf

2. Organización Mundial de la Salud. Neurociencia del consumo y dependencia del consumo de sustancias Psicoactivas". Washigton. [Seriado en internet] 2005 [Citado 10 marzo de 2017]. Disponible en: http://www.msal.gob.ar/images/stories/ bes/graficos/0000000897cnt-2016-10_ neurociencia-consumo-dependencia-sustancias-psicoactivas_resumen.pdf

3. Observatorio de drogas de Colombia. Sustancias psicoactivas. [Seriado en internet] 2006 [Citado10 marzo de 2017]. Disponible en http://www.odc.gov.co/problematica-drogas/consumo-drogas/sustancias-psicoactivas

4. Estudio Nacional de Consumo de Sustancias Psicoactivas en Colombia - 2013. [seriado en internet] 2013 [Citado 10 marzo de 2017]. Disponible en: https://www. unodc.org/documents/colombia/2014/Julio/Estudio_de_Consumo_UNODC.pdf

5. Gutiérrez Quesada M. Drogas y adicciones. definición y características de las drogas. Psicoactiva. [Seriado en internet] [citado 10 marzo de 2017]. Disponible en: https://www.psicoactiva.com/ebook/ ebook-drogas.htm
6. Oficina de las Naciones Unidas contra la Droga y el Delito (UNODC) manifestaron. Informe mundial sobre las drogas. 2015. Resumen ejecutivo. [Seriado en internet] 2015 [Citado 10 marzo de 2017]. Disponible en: https://www.unodc.org/documents/wdr2015/WDR15_ExSum_S.pdf

7. Organización Mundial de la Salud OMS. La dependencia de sustancias es tratable, sostiene un informe de expertos en neurosciencias. [Seriado en internet] 2004 [Citado 10 marzo de 2017]. Disponible en: http://www.who.int/mediacentre/ news/releases/2004/pr18/es/

8. Ley 1122 de 2007: Por la cual se hacen algunas modificaciones en el Sistema General de Seguridad Social en Salud y se dictan otras disposiciones [Internet] Colombia: Ministerio de Salud y Protección Social; 2007[Consultado el 9 de febrero de 2017] Disponible en: https://www.minsalud.gov.co/sites/rid/Lists/BibliotecaDigital/RIDE/DE/DIJ/ley-1122-de-2007.pdf

9. Observatorio de Drogas de Colombia. Situación del consumo de drogas. Ministerio de la Protección Social [Seriado en Internet] Abril 28 de 2017 [Consultado 17 Octubre 2015]. Disponible en: http://www. odc.gov.co/problematica-drogas/consumo-drogas/situacion-consumo

10. Navia-Bueno M, Farah-Bravo J, Yaksic-Feraudy N, Philco-Lima P, Magosso Takayanagui AM. Conocimiento sobre el fenómeno de las drogas entre estudiantes y docentes de la Facultad de Medicina de 
la Universidad Mayor de San Andrés. Rev. Latino-Am. Enfermagem (La Paz Bolivia) mayo-junio 2011;19: 722-729 [Internet] [Consultado 12 de diciembre 2011]. Disponible en: www.eerp.usp.br/rlae

11. Wright MGM, Takayanagui AMM, Ventura CAA. Salud Internacional y el fenómeno de las drogas en las Américas. Dimensiones de las relaciones internacionales en el contexto de la globalización, de la salud internacional y de la internacionalización de riesgos. Ribeirão Preto (SP): Escuela de Enfermería de Ribeirão Preto, SENAD; 2007. Modulo I Parte I, p. $1-39$

12. Melina Castrillo J, De la Cruz Ortiz A, De la Salas Rodríguez K, Eguis Valencia $\mathrm{R}$, Galves Rondón D, Caro de Pallares S, Rosales Amarís M, Cristoph R, Palmet M. Conocimientos, actitudes y prácticas relacionados con el consumo de sustancias psicoactivas en estudiantes de enfermería de una universidad del departamento del Atlántico (Colombia) Revista Científica Salud Uninorte [Seriado en internet] 2012 [Consultado 10 marzo de 2017]. 28 (2): 322-334. Disponible en: http://www.scielo. org.co/pdf/sun/v28n2/v28n2a14.pdf

13. Lastre Amell G, Gaviria García G, Arrieta Reales N. Conocimiento sobre el Consumo de Sustancias Psicoactivas en Estudiantes de Enfermería. Revista Ciencia e Innovación en Salud. 2013;1(1): 46-51

14. Castellón H, Rocha C, Montes L, Lastre G. Conocimientos, actitudes y prácticas del consumo de sustancias psicoactivas en estudiantes de enfermería de una Universidad privada de BarranquiIla 20102011. Respuestas. 2015; 20(1): 67-83.

15. Resolución 8430 de 1993: Por la cual se establecen las normas científicas, técnicas y administrativas para la investigación en salud [Internet] Colombia: Ministe-

70 rio de Salud y Protección Social; octubre 4 de 1993 [Consultado marzo 10 de 2017]. Disponible en: https://www.minsalud.gov.
co/sites/rid/Lists/BibliotecaDigital/RIDE/ DE/DIJ/RESOLUCION-8430-DE-1993. PDF

16. Estudio Nacional de Consumo de Sustancias Psicoactivas en Colombia - 2013. [Seriado en internet] 2013 [Consultado 10 marzo de 2017]. Disponible en: https://www.unodc.org/documents/ colombia/2014/Julio/Estudio_de_Consumo_UNODC.pdf

17. CoullautValera R, Arbaiza Díaz del Río I, De Arrúe Ruiloba R, Coullaut Valera J, Bajo Bretón R. Deterioro cognitivo asociado al consumo de diferentes sustancias psicoactivas. Actas Esp Psiquiatr. 2011;39(3):168-173

18. Barbieri I, Trivelloni M, Zani B, Palacios $X$. Consumo de sustancias psicoactivas en los contextos recreativos entre estudiantes universitarios en Colombia. Revista Ciencias de la Salud 2012. [Seriado en internet] [Consultado 10 marzo de 2017] 2012:1069-86 Disponible en: http://www. redalyc.org/articulo.oa?id=56223337007

19. Moral MV, Rodríguez Francisco J, Ovejero A. Correlatos psicosociales del consumo de sustancias psicoactivas en adolescentes españoles. Salud pública Méx [Revista en la Internet] [Consultado 10 marzo 2017] 2010;52(5):406-415. Disponible en: http://www.redalyc.org/articulo.oa? id $=10617417008$

20. Hernández M, Sánchez D, Cañón $Y$, Rojas J, León A, Santos M. Factores de riesgo psicosociales que inciden en el consumo de sustancias psicoactivas en los adolescentes. Revista Línea de Vida. [Seriado en internet] [Citado 10 marzo de 2017] 2015; 24-32. Disponible en: http:// revia.areandina.edu.co/ojs/index.php/Kk/ article/view/370 